\title{
EN RECUERDOS DE ADOBE. EVALUACIÓN Y PUESTA EN VALOR DE LAS CASAS PATRIMONIO DE ADOBE EN MASAYA, NICARAGUA ${ }^{(*)}$
}

\author{
ADOBE MEMORIES. ASSESSMENT AND VALUE ENHANCEMENT \\ OF HERITAGE ADOBE HOUSES IN MASAYA, NICARAGUA \\ NOHEMÍ BALMACEDA PUTOY ${ }^{(\star x]}$
}

Fecha de recepción: 30 de agosto de 2016

Fecha de aprobación: 26 de noviembre de 2016

\begin{abstract}
RESUMEN
Este artículo trata de la importancia de las casas patrimonio, principalmente la casa de Ana María Vega donde habita la familia Fariñas, y propone que los elementos esenciales no son solo la fachada y el entorno. Una vivienda va más allá de su ubicación, su entorno, su estética, y su forma, incluso si el entorno está en revolución, como lo expone Kevin Lynch, quien afirma que el entorno influye en la asimilación o el rechazo de un edificio. Asimismo, cualquier tipo de intervención debe estar pensada desde el usuario, pero no el que circula por la calle y entra en contacto solo visualmente con el lugar, sino con el que convive con esas paredes, el que desarrolla sus historias y sus vivencias tras el adobe. Esto se debe a que este artículo se enfoca en el interior; lo que toma en cuenta que no podemos saber a dónde vamos si no sabemos de dónde venimos.
\end{abstract}

\section{PALABRAS CLAVE}

Masaya-Nicaragua, conservación del patrimonio edificado, casas de adobe

\section{ABSTRACT}

This article is based on the importance of heritage houses, mainly in the house of Ana Maria Vega Fariñas, where the Fariñas family lives. It puts forward that what matters is not only the facade and the environment: a house as a whole goes beyond its location, environment, aesthetics and form, and is always in movement. It is Kevin Lynch who says that the environment influences the assimilation or rejection of a building. Also, any intervention must be designed from the point of view of the user. This point of view should include more than just those who circulate the streets and comes into contact with this place only visually. It should also take into account those who coexist with those walls, who tell their stories and live their lives behind the adobe. This article focuses on the indoor experience; it takes into account that we do not know where we are going but we know where we are coming from.

\section{KEYWORDS}

Masaya-Nicaragua, preservation of built heritage, adobe houses

(*) El presente artículo forma parte del trabajo de investigación elaborado en el marco del curso de grado Metodología de la Investigación (2015-actualidad), que es guiado por el tutor MSc. Arq. Myrna Mendoza. Ha sido presentado ante el jurado en la XXXI Jornada Universitaria de Desarrollo Científico (JUDC), donde obtuvo el primer lugar en su rama. Se ha ampliado en el tema, que en inicio núcleo de un ensayo de evaluación urbano arquitectónica del área patrimonial de la ciudad de Masaya (Propuesta de imagen urbana patrimonial en el casco antiguo de Masaya en las avenidas San Juan y Real de Monimbó). Este tema será retomado para la obtención del título de arquitecto en la Universidad Nacional Autónoma de Nicaragua (UNAN-Managua).

$\left.{ }^{(*}\right)$ Egresada de la Facultad de Arquitectura de la Universidad Nacional Autónoma de Nicaragua (UNAN-Managua). Su ciudad natal es Masaya-Nicaragua, donde desarrolló una pasantía en el Área de Catastro de la Municipalidad. Ha colaborado como voluntaria en la evaluación de daños de la arquitectura religiosa de la ciudad de León y ha realizado la inspección de viviendas populares. Obtuvo el primer lugar en la categoría de ensayo en la XXXIV JUDC de la UNAN. Contacto: nohemi952@yahoo.es 
Las memorias de las personas muchas veces desparecen con ellas, pero la arquitectura es un testigo silencioso de la historia y el mejor ejemplo del acervo cultural que distingue a una ciudad de otra.

Nicaragua posee ciudades que son consideradas importantes íconos patrimoniales por la enorme cantidad de inmuebles, así como por su cultura. Entre las más representativas se encuentran ciudades como Granada y León, y esto se debe en gran parte a que Nicaragua tiene una normativa que ayuda a intervenir en la restauración y la conservación, pero existen otras ciudades con inmuebles muy importantes como Matagalpa o Masaya (Balmaceda, 2016).

La evaluación es un tema que no debe tomarse a la ligera, más si se entiende que la información evaluada permite descubrir la identidad de un inmueble, una ciudad, un grupo de personas y un país. Esta alcanza más importancia cuando hablamos de patrimonio y su estado de conservación.

La documentación de patrimonio urbano arquitectónico de Masaya es escasa, por lo cual debe recurrirse a trabajos monográficos y tesinas, a los inventarios de bienes culturales inmuebles del Instituto de Cultura (INC), a las fichas resumen del patrimonio construido del inventario nacional de bienes culturales, a la propuesta de normativa de Masaya, principalmente, y a las leyes a nivel nacional. Estas toman de referencia a León y Granada; sus ordenanzas municipales son la guía nacional. También las leyes de países vecinos como Honduras y Guatemala se aprovechan para reconocer el gran valor que posee la conservación del patrimonio en una ciudad como Masaya.

La documentación y la correcta evaluación del estado de conservación permitirán conocer e identificar el valor del inmueble, no solo a nivel estético-formal, monumental e histórico, sino también económico; este tipo de instrumento permitirá a la alcaldía, así como al INC y el Instituto de Turismo (INTUR), tener a su disposición un sistema de información del patrimonio local en Masaya (Balmaceda, 2015).

En Nicaragua hay dos entidades que se encargan de velar por el patrimonio: el INC y la Dirección de Patrimonio Cultural.

EI INC es la entidad rectora del sector cultural nicaragüense: tiene como objetivo formular, coordinar, ejecutar y vigilar la política del Estado en materia cultural. Incorpora en su presupuesto anual proyectos de restauración y puesta en valor de bienes culturales inmuebles bajo su administración. La Dirección de Patrimonio Cultural, constituida por varios departamentos de acuerdo al tipo de bienes culturales, ejecuta las directrices del INC en materia de conservación, preservación, investigación, difusión y puesta en valor del acervo cultural de Nicaragua. Para el caso específico de los Bienes Culturales Inmuebles, cuenta con el Departamento de Conservación Urbana y Edilicia, que se encarga de regular y normar las intervenciones en este tipo de bienes culturales. Para realizar esa labor recibe apoyo de las alcaldías de León, y Granada, compuesta por el Departamento de Registro Nacional de Bienes Culturales, el Departamento de las Redes de Protección al Patrimonio Cultural, el Departamento de Arqueología, el Departamento de Tráfico llícito, y el Departamento de Normas y Procedimientos.

\section{Masaya y su historia en casas de adobe}

Masaya es una ciudad que se caracteriza por sus invaluables manifestaciones culturales y los importantes acontecimientos históricos que han construido a lo largo del tiempo su identidad. Una ciudad con estructura social, que debe responder a las diversas necesidades que cada época le plantea, que busca la combinación armónica de lo nuevo con lo viejo y de lo moderno con lo tradicional; así escribe su historia, y transmite su cultura y folklore. De esta manera, se asegura la perdurabilidad de las tradiciones y costumbres que en ella afloran y se transmiten. 

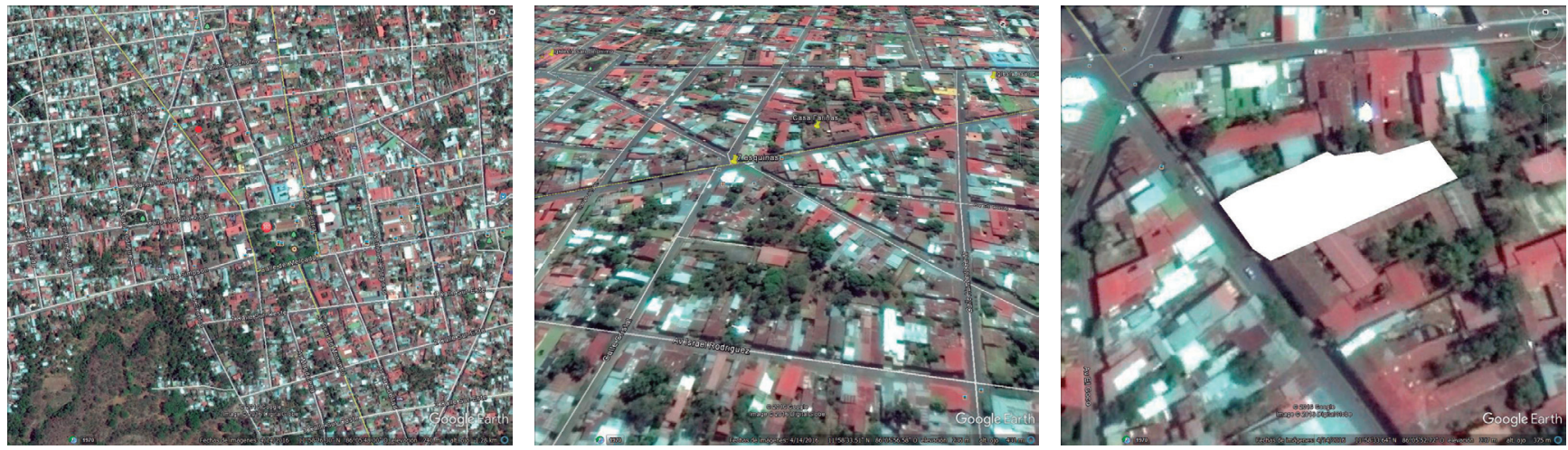

La ciudad de Masaya es de las más antiguas e importantes del país; se destaca por la disposición de su núcleo urbano más antiguo y la manera en la que este se relaciona con los demás núcleos urbanos. En su conjunto, la ciudad posee un gran valor urbanístico, arquitectónico e histórico, en especial es valiosa la vinculación con el barrio indígena de Monimbó. Además, se caracteriza por su amplia actividad productiva y es considerablemente reconocida a nivel nacional por su industria artesanal (Secretaría Técnica de la Alcaldía de las Familias y Comunidades de Masaya, 2012).

Masaya posee una línea urbana arquitectónica con alto valor patrimonial. Entre los inmuebles que la componen se encuentra la casa de la familia Fariñas. En la actualidad muchas personas no se preocupan por el patrimonio; algunos arquitectos incluso lo consideran algo pasado de moda. Sin embargo, la historia no pasa de moda, puesto que es relativa: lo que hoy es presente, mañana será historia. El patrimonio debería ser considerado de suma importancia, pero lo cierto es que muchas veces incluso las municipalidades, al no tener una normativa vigente, abandonan el patrimonio por el progreso y las nuevas tendencias constructivas.

En una calle de Masaya, una de las más congestionadas de la ciudad, pues es una de las que conforman las siete esquinas, una confluencia de calles que unen ejes rectores, lo que la convierte en un gran nodo. Cerca de aquí se encuentra la casa de la familia Fariñas.

\section{Las famosas Siete Esquinas}

En Masaya, la confluencia de tres caminos, de cuyos orígenes únicamente nos podrían relatar la historia, son el vestigio de primitivas comunidades que habitaron la zona y transitaron esas rutas, y que con sus huellas heredaron a sus sucesores accidentes viales raros, pero seguros.

La mayoría de calles, callejones, pasajes, plazas, tiangues, templos y parques de Masaya conservan el sello ancestral. Las principales vías son angostas, y, en general, salvo algunas, son cortas, ya que la planicie de la primitiva ciudad tenía limitaciones naturales. Durante el proceso de desarrollo de los últimos 40 años, los obstáculos salvables en períodos de expansión fueron la pauta para la construcción de pistas modernas. La urbe se podía extender hacia el Noroeste y Noreste, y, limitadamente, también hacia el Oeste; por la laguna, que es un limitante, era imposible expandir hacia la zona de Monimbó (Figuras 1 y 2 ).

Entre las comunidades de las iglesias San Jerónimo y San Juan, se encuentra el fenómeno vial que el pueblo bautizó como Siete Esquinas. Ese cruce está formado desde época indeterminada por la necesidad de tránsito de los habitantes de Monimbó y Nindirí, quienes establecieron un camino que se cruzó con el que existía antes. Viajeros
Figura 1. Foto aérea de Masaya y ubicación de Siete Esquinas

Fuente: Elaboración propia a partir de fotografías de Google Earth, 2016. 
devenir Vol. 3, Nº, JULIO- DICIEMBRE 2016, PP. 49-62 - EstUDIOS I ISSN 2312-7570

UNIVERSIDAD NACIONAL DE INGENIERÍA, LIMA

Figura 2. Plano de Masaya y ubicación de la casa Fariñas

Fuente: Archivo fotográfico Nohemí Balmaceda, 2015.

Figura 3. Vista de las famosas Siete Esquinas

Fuente: https://www.youtube.com/ watch?v=Dhqp5j-ZSBU, 16/10/2011, captura de pantalla, 2016.

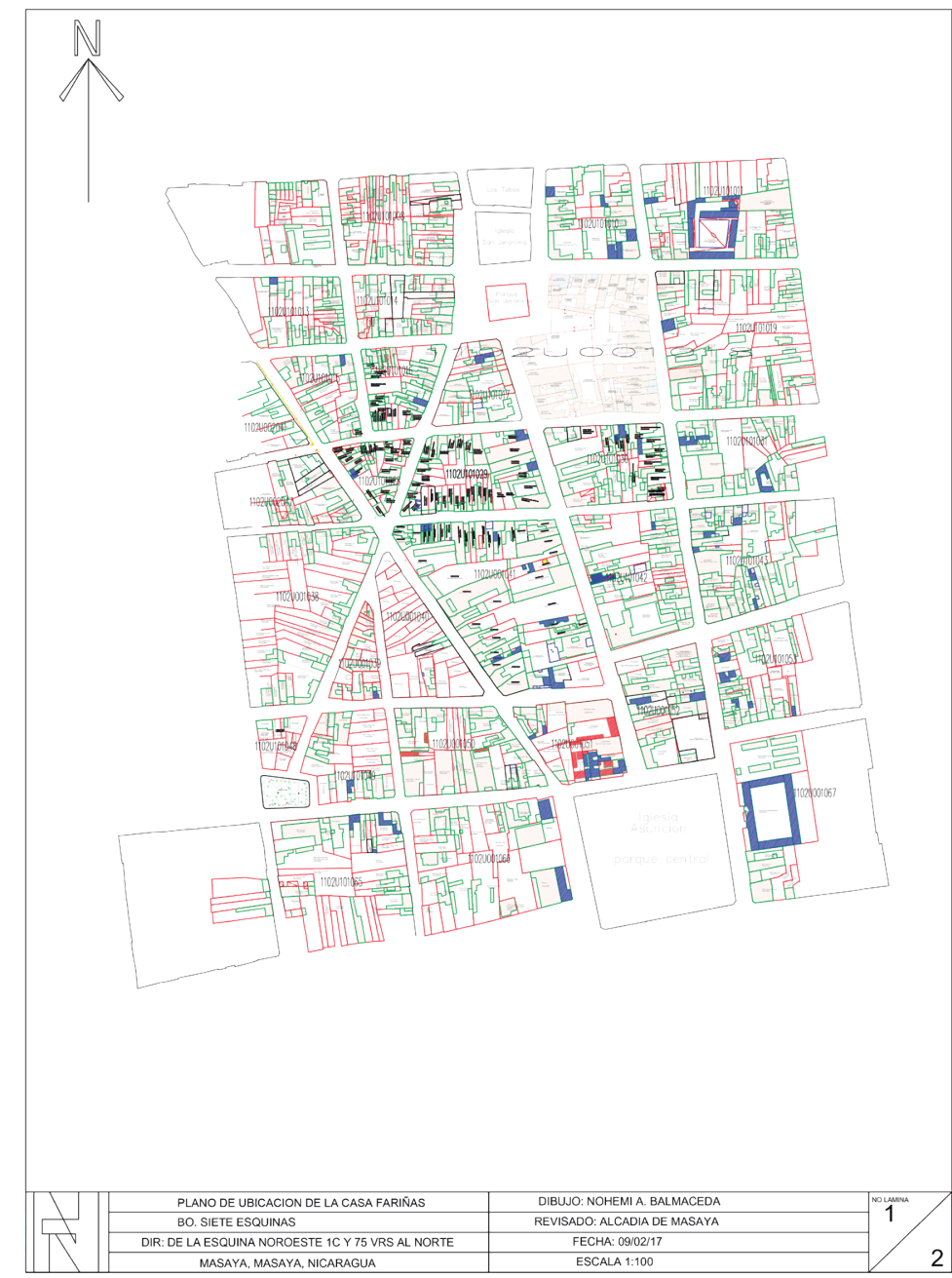

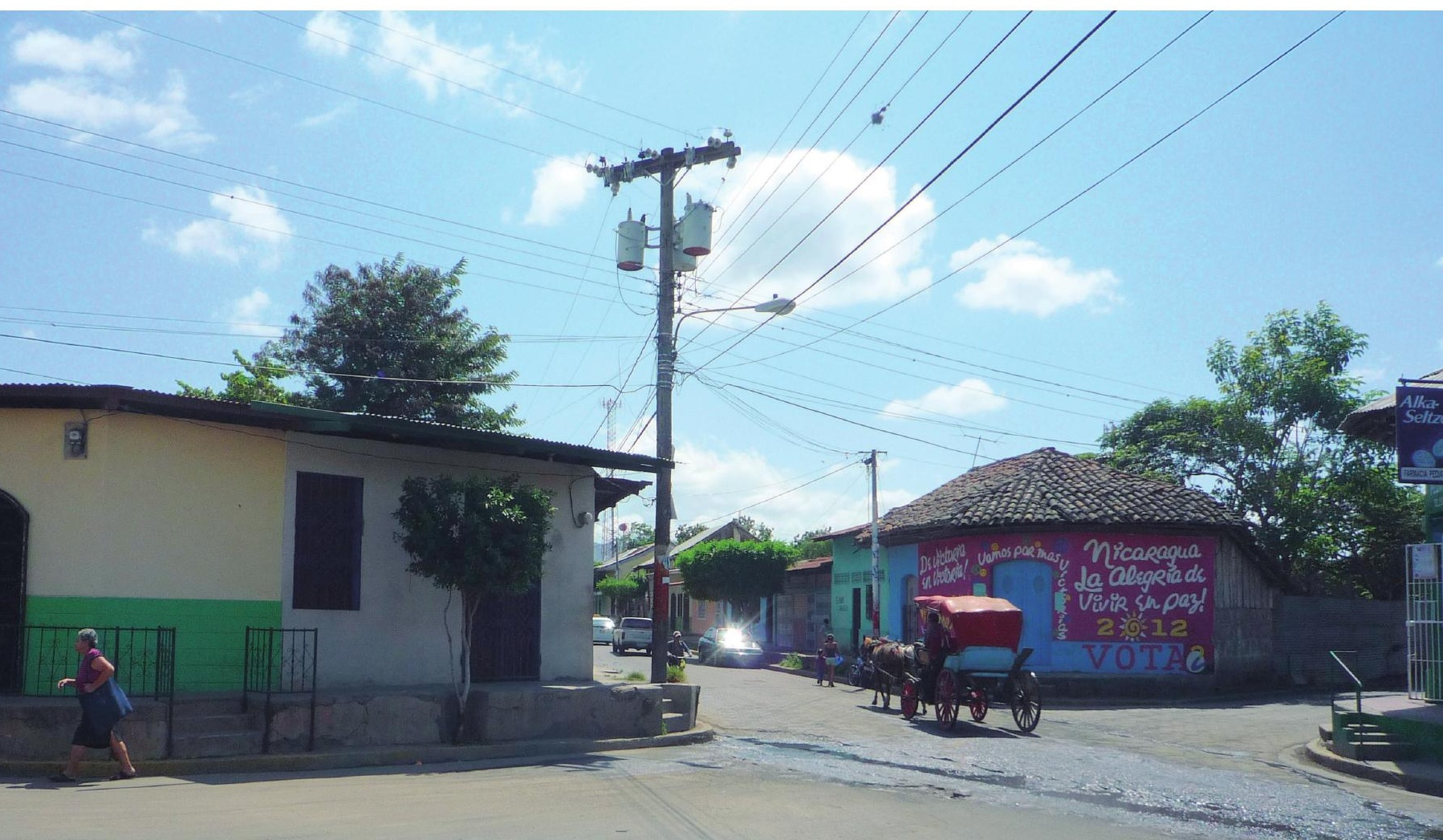


procedentes del Noreste y Este de la zona, y posiblemente de más allá -al parecer al acarrear agua, pescar y recolectar alimentos de la laguna- necesariamente surcaron esos caminos, bifurcaron otros atajos y los configuraron según sus destinos (Las Famosas Siete Esquinas, 2009) (Figura 3).

La casa de la Familia Fariñas, que a pesar del tiempo y su estado de conservación alberga dentro recuerdos del Gran Hotel Azcárate, parte de su lobby con el piso original, las puertas con vasistas y la división original con sus paredes de adobe. Estas ahora están muy maltratadas, debido al paso del tiempo y al poco o ningún mantenimiento que le ha dado esta familia de escasos recursos, de la que forma parte el señor Fariñas, que trabaja como maestro de obra, pero que aún con su poco nivel escolar entiende el valor de su casa y destaca el valor estético de la misma, así como de las demás construcciones de adobe (Figura 4).

\section{Puertas}

Las puertas, al igual que los portones y las ventanas, están hechas acorde al estilo original del edificio; por ello, forman parte primordial de su imagen. Como se aprecia con facilidad en la casa Fariñas, el gusto y sobre todo la economía de las personas que la habitan son los factores que determinan los diferentes estilos, los tamaños e incluso los acabados de las puertas (Orías, 2011).

Por ello la carpintería es tan valiosa como el resto de los elementos que conforman la identidad de una casa, y en este caso uno de los elementos que hace tan particular su imagen y evidente su data a simple vista. Este edificio que ahora se encuentra en estado de alto deterioro fue el escenario de grandes reuniones de la elite de Masaya en los tiempos del hotel. El diseño sencillo de una puerta no la convierte en menos valiosa que una con más elaboración; por el contrario, la simpleza de esta es un claro indicio de mayor antigüedad, por lo cual resulta más preciada. Las puertas, durante la Colonia, estaban formadas con tablones; ya en el barroco eran mucho más elaboradas, decoradas con tallados vegetales y curvilíneos, un estilo más dado a los edificios civiles y religiosos. En las casas coloniales son comunes las mirillas o vasistas.

La calidad de las puertas, que han sido testigos supervivientes de guerras, terremotos y algún otro suceso natural, es evidente a primera vista. Estas puertas, centinelas de la seguridad de la familia, se encuentran en alto estado de deterioro por la falta de mantenimiento y los efectos de la humedad. Lo fascinante es el hecho de que, a pesar de la carestía de recursos, sus habitantes no hayan tomado la arbitraria decisión de destruir este bien; por el contrario, comprenden la riqueza estética con la que cuenta su inmueble. Las puertas, al ser elementos muy vulnerables en las obras de reforma o rehabilitación, suelen ser desestimadas y sustituidas. Este proceder es totalmente errado, puesto que los materiales y técnicas empleados en su elaboración las convierte en inesperadas obras de arte que deben ser conservadas. Para ello deben pasar por un proceso en el que, en primera instancia, se eliminen los factores que las deterioran, como una medida preventiva antes de avanzar con las intervenciones.

\section{Protección de las carpinterías}

Los productos protectores y desinfectantes son adaptables a las carpinterías históricas, como las soluciones acuosas y orgánicas ventajosamente protectoras, entre estos el arseniato de cobre, el cloruro de zinc cromado y aquellas de boro, como el cloruro de boro cromado y el boro de zinc cromado -estos dos últimos con propiedades retardantes de fuego-. Entre los protectores de disolvente orgánico, se citan: Ios fenoles clorados, esteres de boro, estaños tribulíticos y compuestos de amonio cuaternario. El paradiclorobenceno es el producto más utilizado en el medio, pero debe aplicarse con precaución debido a su alta toxicidad, por lo cual en este trabajo se emplean gafas de protección, máscaras y guantes, y es realizado por especialistas (Orías, 2011).
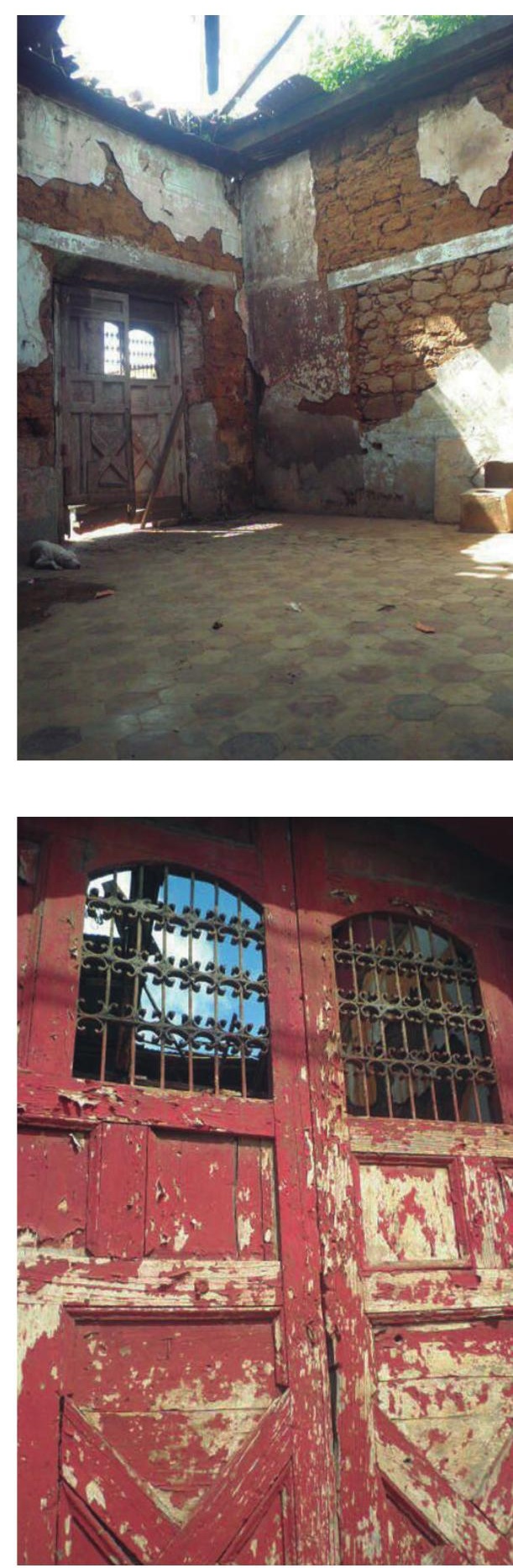

Figura 4. Antiguo lobby del hotel Fuente: Archivo fotográfico Nohemí Balmaceda, 2015.

Figura 5. Puerta principal donde se aprecia el deterioro y el desgaste de la puerta y sus vasistas

Fuente: Archivo fotográfico Nohemí Balmaceda, 2015 

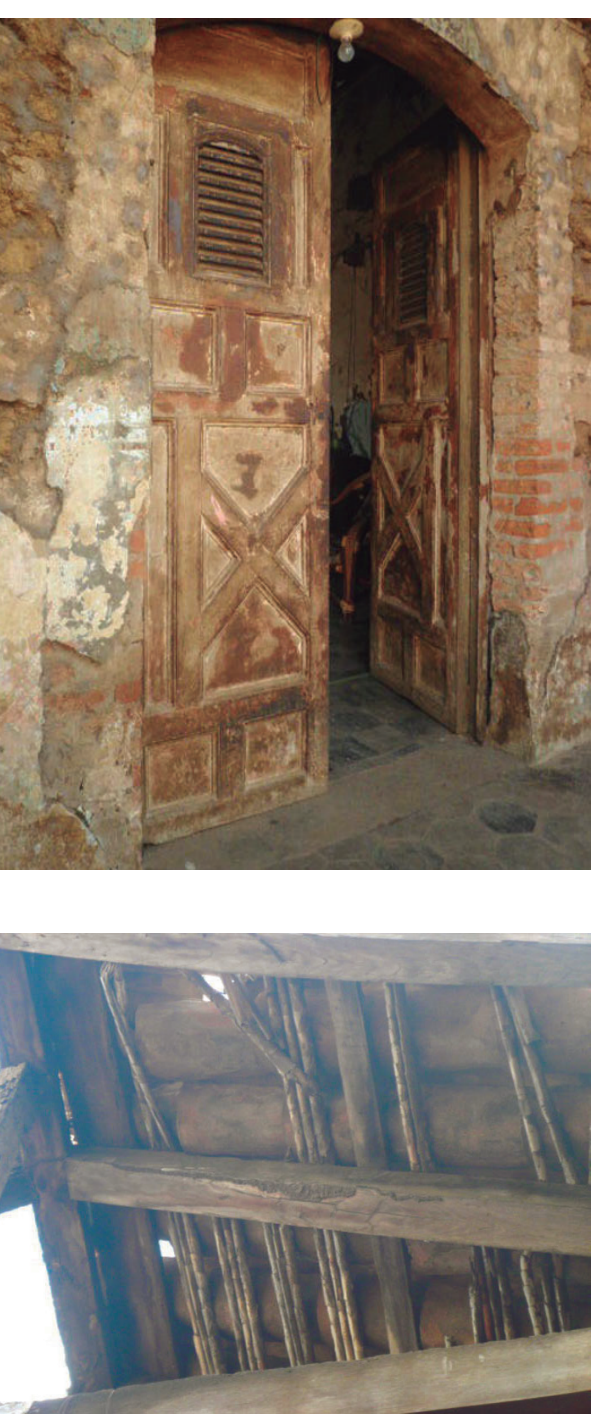

Figura 6. Puertas internas de la casa de la familia Fariñas, que han sido mantenidas con su estructura original y el tinte original, sin ninguna especie de cubierta de pintura

Fuente: Archivo fotográfico Nohemí Balmaceda, 2015

Figura 7. Vista del armazón del alero con cañahueca y madera

Fuente: Archivo fotográfico Nohemí Balmaceda, 2015.
Sin embargo, un correcto procedimiento en la conservación de las carpinterías de madera debe documentar las piezas a tratar, valiéndose para ello de fotografías y croquis. Asimismo, son de gran ayuda las fotografías antiguas en caso de reponer faltantes, aunque estas puertas, a pesar de su alto estado de deterioro por humedad, tienen sus piezas completas (Figura 5).

La limpieza debe ejecutarse manualmente con herramientas que no dañen la madera o con productos químicos (decapantes), por lo cual el artesano debe estar debidamente protegido. Posteriormente, se elimina los posibles agentes bióticos con un preparado de aceite de linaza y desinfectante. En caso existan piezas sueltas, se procederá a una consolidación inmediata mediante pegamentos, prensas y clavos sin cabeza. Cuando se deba reponer una pieza perdida se deberá utilizar el mismo tipo de madera que hay en la carpintería original, y se ensamblaran con la ayuda de cajeados y llaves. Es muy importante rehidratar las paredes antes de otorgarles el acabado final, para lo que se utiliza aceite de linaza. Es necesario que la pieza seque varios días en un ambiente aislado de polvo, ya que el aceite de linaza húmedo mezclado con el polvo produce una placa antiestética y difícil de remover.

Después de consolidar y restituir sus características originales, se procede a la protección con barnices poliuretanos incoloros satinados que contengan filtro ultravioleta, porque este producto otorga un acabado de encerado natural y ofrece una protección duradera. Para nada es aconsejable pintar las puertas, lo que es muy notorio no solo en las puertas de la casa de la familia Fariñas, sino en casi todas las puertas de la ciudad, principalmente en muchas de las casas patrimonio. Tiene el fin de tapar ciertas imperfecciones, pero lejos de cumplir ese objetivo deja más en evidencia la problemática de las mismas. Es más conveniente lograr una textura homogénea utilizando tintes que unifiquen las reposiciones y consolidaciones con la madera original (Figura 6).

\section{Los aleros}

Los aleros de las casas tradicionales responden a diversos estilos y formas que, por lo general, corresponden a inmuebles construidos en la época colonial, aunque también a la arquitectura vernácula o popular; son muy distintas las casas que optan por los parapetos. Los aleros tradicionales están formados por la superposición decorativa de una hilera de tejas y ladrillos encasillados, o por la prolongación de la estructura al exterior con cañahueca o entablado superior con las tejas voladas (Orías, 2011). Sin embargo, siempre están sobre el muro de la calle o al interior, como en los corredores que forman las arcadas alrededor del patio interno de la casa Fariñas con los canecillos terminados en moldura. Estos son totalmente típicos de las casas; a menudo son sustituidos por aleros enlucidos de menor calidad (Figura 7).

\section{Cielo raso}

En las antiguas casonas de Masaya el cielo raso también se convertía en centro de atención por su detallada decoración y ornamentación; en la casa de la familia Fariñas, aunque no está conservado en su totalidad, aún se conserva parte de él. Se encuentra en lo que anteriormente hubiese sido el lobby del hotel con un estilo más minimalista que el de otras viviendas pero igual de estético. Es una lástima que no exista, como en otros países de Latinoamérica, un subsidio gubernamental para rescatar estas piezas icónicas de la historia y la cultura de la ciudad, porque el patrimonio define sin palabras el acervo cultural de una ciudad o de una nación. Incluso cuando la política sí trata el tema de turismo, deja por fuera el patrimonio, como si no fuera parte de la cultura tangible, o como si la arquitectura por estar en estado de deterioro no valiese lo mismo que una artesanía o la gastronomía (Figura 8).

De nuevo, la madera, al ser un material orgánico, es fácilmente atacable por agentes destructores, principalmente los biológicos. Esto es lo que sucede en la casa de la fa- 
milia Fariñas, donde, debido a la exposición a la intemperie, se encuentra destruido el cielo raso y es recomendable realizar una restauración. Además, por el estado en el que se encuentra la madera no hay más remedio que sustituirla; ya es demasiado tarde para reintegrarla, el otro procedimiento aceptable, por el nivel de deterioro. Sin embargo, en algunas ocasiones los elementos de madera aún conservan sana una parte significativa, y es posible restablecerles su capacidad de trabajo mecánico; sin embargo, otras veces, cuando una parte es insustituible por su valor estético, histórico o artístico, se sabe que lo requerido es la consolidación (Figura 9).

\section{Fallas en la madera}

Existen diferentes fallas en la madera, ya sean estructurales u ornamentales, entre ellas la alteración del material por la humedad que afecta la cabeza de las vigas que se puede observar en el techo de lo que era el lobby del antiguo hotel, la zona más expuesta. Esto ocurre sobre todo si el muro de apoyo es de cerramiento o si existen canalizaciones de aguas próximas; entonces, la estructura del techo se vuelve peligrosa, como en el caso de la casa de la familia Fariñas, donde ha alcanzado niveles de putrefacción. También son un factor de deterioro las termitas que atacan las cabezas, lugar por el que ingresan a través del muro; la humedad solo ayuda a la actuación de las termitas, razón por la cual es normal encontrar ambos fenómenos a la vez, mayormente en las áreas humedad como baños, cocinas, y bajantes. En el caso de la casa de la familia Fariñas, lo provocó el mal estado de la cubierta de techo. Se sabe que el fuego y la intemperie son otros factores que deterioran la madera, y eso explica que no solo el cielo raso esté deeteriorado, sino también las puertas y las ventanas (Orías, 2011).

Otro factor son las lesiones mecánicas, que ocurre cuando una pieza pierde su función como elemento resistente, lo cual puede observarse en el cielo raso de la casa de la familia Fariñas que está fracturado y deformado por el exceso de cargas de viento y agua, así como la carga muerta propia de la estructura. Este efecto, por supuesto, es mayor a la capacidad de trabajo para la cual fue diseñada la pieza y provoca una leve pendiente al interior de la casa.

Resulta incuestionable que, si se proyecta conservar los valores estéticos, urbanos, arquitectónicos que conforman la singularidad de las casas de Masaya, no se debe desatender a las cubiertas, parte sustancial de su imagen. El hecho de que esté en la parte más alta del edificio no implica que deba pasar inadvertida; al contrario, es de gran importancia porque contribuye a la imagen completa del edificio, y se debe tener extrema mesura al intervenirlas.

\section{Las cubiertas de techo}

Por lo general, las cubiertas de las casas de estilo colonial son de teja, pero los dueños de la mayoría de los inmuebles han decidido cambiarlas por zinc o por láminas plásticas traslucidas para aportar mayor luz a la vivienda. En la casa Fariñas se aprecia solo un cambio en el extremo norte del corredor, donde este se junta con el muro de la propiedad vecina (Figura 10).

Las cubiertas están constituidas por dos elementos básicos, la armazón y el tejado, que en estos casos corresponden a estructuras de madera, ya sea labrada o simple, y tejas cerámicas curvas Ilamadas en muchas localidades 'coloniales', aunque en realidad no pertenecen al tiempo de la Colonia y muchas veces son falsos históricos (Figura 11).

En este estilo de arquitectura, el tejado fue concebido con un tipo de cubierta que cumplía no solo funciones aislantes frente a las inclemencias del clima -la temperatura, principalmente-, sino también funciones estéticas; de ahí que la primera premisa sea la de consolidar la imagen por medio del mantenimiento de los tejados en perfecto estado con las intervenciones correctas. Entre los trabajos rutinarios de mantenimien-
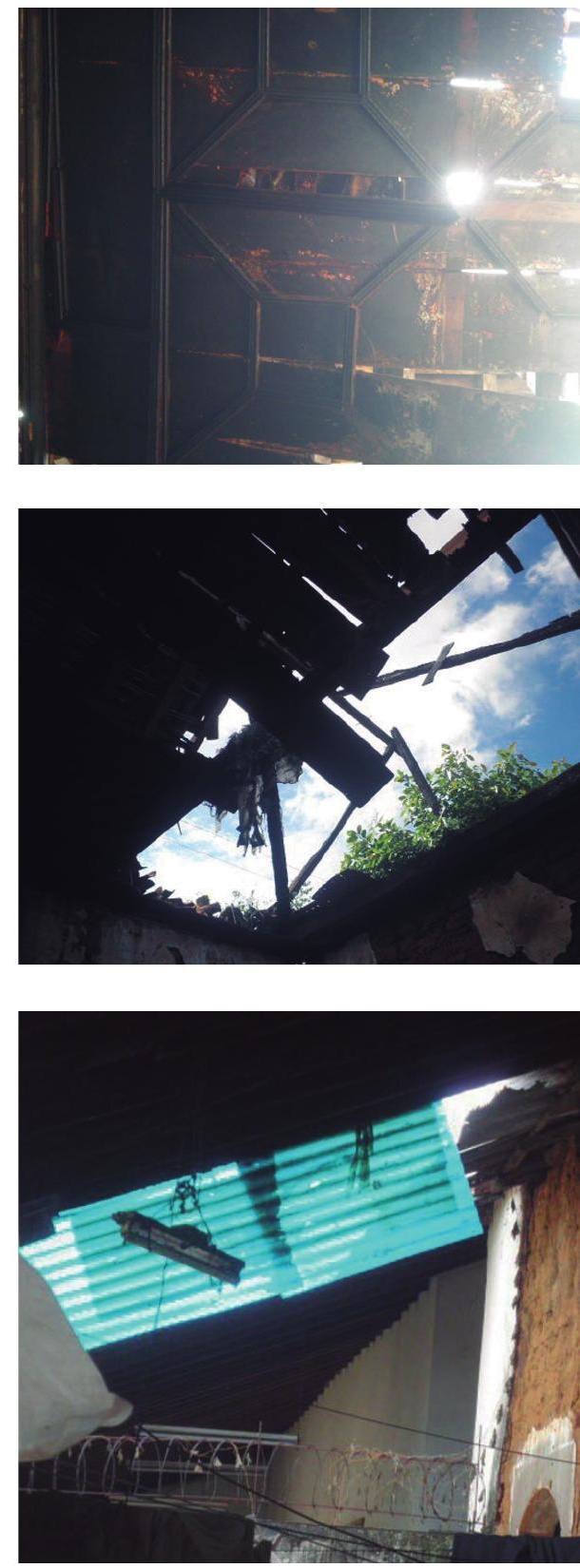

Figura 8. Vista del cielo raso en el lobby del antiguo Hotel Azcárate

Fuente: Archivo fotográfico Nohemí Balmaceda, 2015.

Figura 9. Detalle del cielo raso con líneas puristas formadas por cuadrados unidos por líneas diagonales en madera color chocolate

Fuente: Archivo fotográfico Nohemí Balmaceda, 2015.

Figura 10. Área del techo que fue reemplazada por láminas traslúcidas Fuente: Archivo fotográfico Nohemí Balmaceda, 2015. 


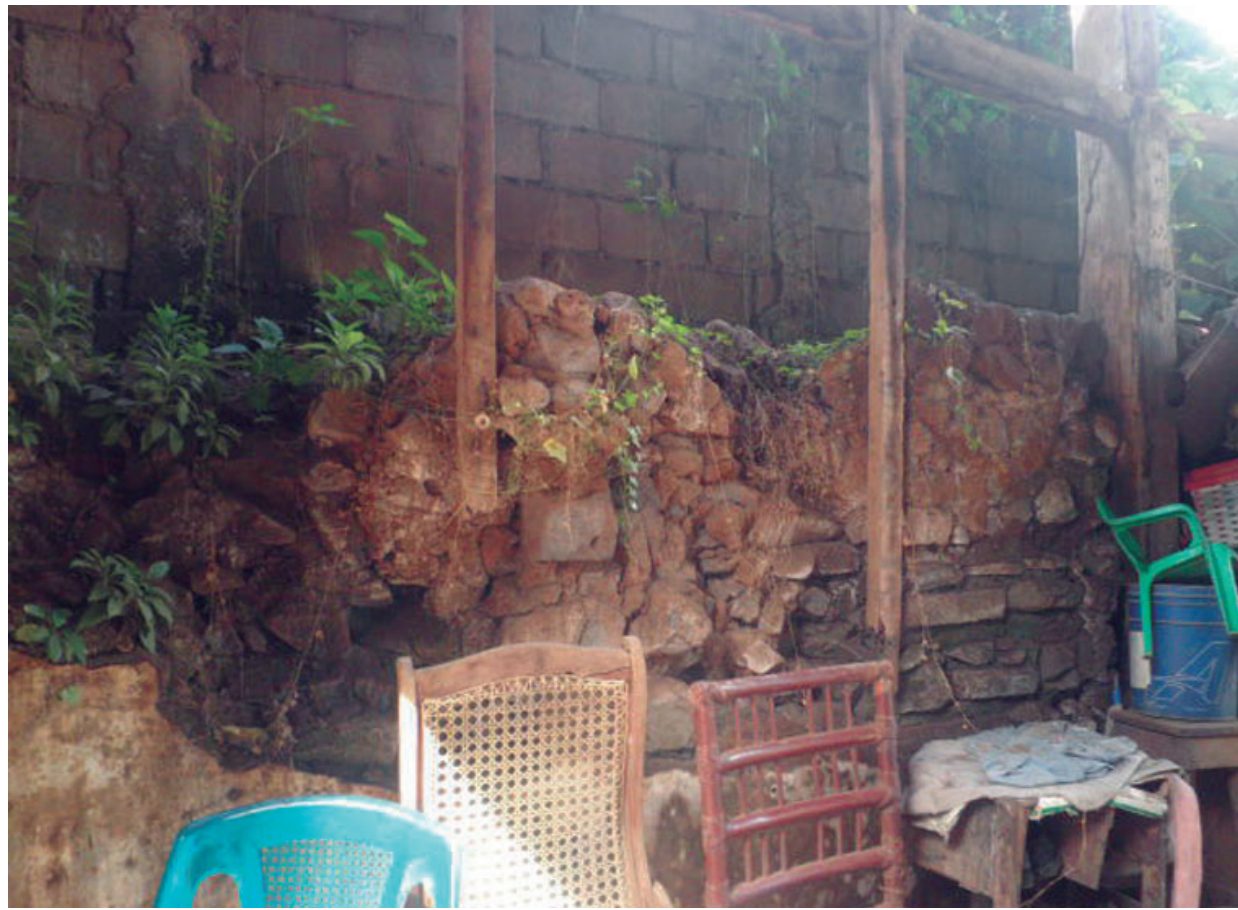

tiene propiedades resistentes al agua En estos casos el agua disuelve el aglomerante de cal y, como se dice popularmente, se va 'lavando' el revoque (Orías, 2011).

Es muy frecuente encontrar vegetación inferior, hongos, musgos y líquenes, que atacan los revoques; al igual que la vegetación parasitaria, que devasta los revoques con raíces que, a pesar de no ser subterráneas, causan desprendimiento. Por otro lado, las aves, en específico las palomas, dañan la cal y el yeso por la gran cantidad de ácido que contiene su excremento, disuelto en agua. Además, al evaporarse el agua, las sales se cristalizan y producen en el revoque las manchas blancas conocidas como salitre. Estas sales se pueden dividir en diversos tipos: sulfatos, cloruros, sulfuros, entre otros (Figura 12).

\section{Muros al exterior}

En los muros que dan al patio se observa el crecimiento de pequeñas plantas que han ocasionado el desprendimiento en los muros, además del fraccionamiento de los bloques de adobe (Figura 13).

Los agrietamientos permiten la entrada de agua hasta las capas interiores, lo que se observa en la Figura 11; esto también produce el desprendimiento y el fraccionamiento de los muros.

Para cada parte de la estructura de una casa patrimonial existen criterios de intervención; los revoques no son la excepción. Para darles mantenimiento se debe proceder, en primer lugar, a limpiarlos, eliminando el polvo, suciedad, afiches y grafitis; para esto se debe utilizar herramientas que no dañen la superficie (lijas suaves y/o espátulas). Del mismo modo, se debe eliminar todos aquellos parches hechos con mortero de cemento, dado que no es compatible con el adobe, $y$, posteriormente, se debe sustituir los parches faltantes con mortero de cal y arena, dando siempre un acabado prolijo; más adelante, se debe sellar con una capa de pintura al agua o la tradicional lechada de cal. Pero si la superficie presenta agentes bióticos, se debe realizar una desinfección con insecticidas y

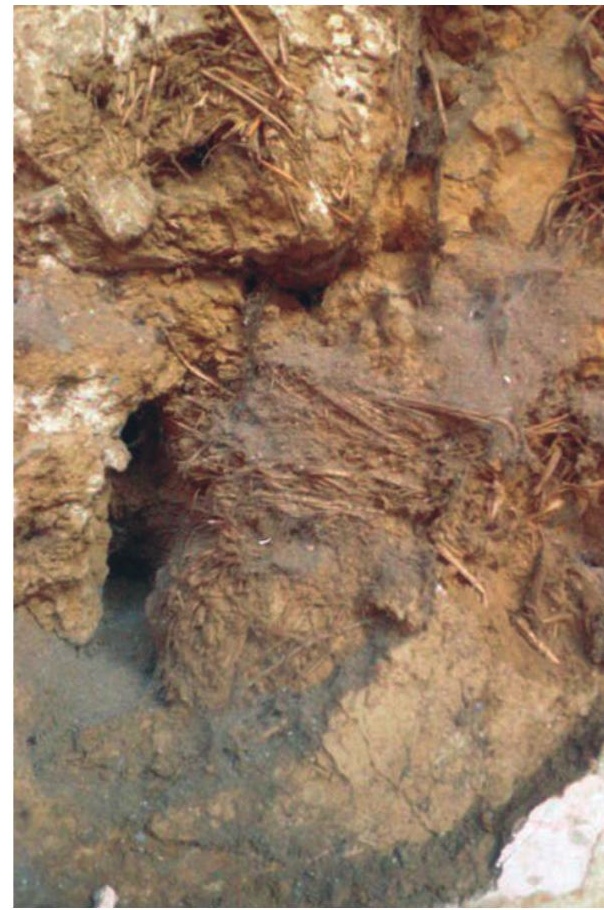

Figura 13. Pared fraccionada y armazón de madera original que ha quedado expuesto como consecuencia de las diferentes patologías que atacaron a estos muros

Fuente: Archivo fotográfico Nohemí Balmaceda, 2015.

Figura 14. Estado de las paredes donde el revoque está demasiado deteriorado para la limpieza, lo que evidencia la necesidad otro tipo de intervención

Fuente: Archivo fotográfico Nohemí Balmaceda, 2015. 
biocidas; de esta manera se evita cualquier propagación. En la casa Fariñas debe limpiarse por el exterior puesto que el interior necesita más trabajo que solo limpieza (Figura 14).

\section{Intervención en revoques}

La intervención apropiada para los revoques en la casa Fariñas podría ser la reparación que se aplica a veces cuando el revoque ha sufrido desconchados o desprendimientos. El primer paso es eliminar todas las artes sueltas y remplazarlas con morteros de las mismas características que las originales -es decir, a base de barro y cal-. Para añadir más adherencia al muro se utilizan técnicas que parten de trabajar mallas y tejas rotas; además, en algunos casos es necesario realizar una eliminación superficial de las juntas de adobe para obtener una mejor unión. Pero la mejor opción, según algunos trabajadores expertos en la materia, es la cal apagada, que aún se encuentra en el mercado y tiene un precio factible. Aún se utiliza la técnica en intervenciones de restauración de gran envergadura, donde se reserva para el apagado de la cal un espacio, piscina o depósito donde se almacena por tres meses antes de su aplicación, y llega a tener una resistencia y un acabado insuperables (Orías, 2011).

Claro que no todas las paredes necesitan una reparación; algunas solo necesitan una consolidación. Esta se recomienda cuando el revoque tiene valor histórico, ornamental, estético, arquitectónico y -no menos importante- urbanístico, como en el caso de los esgrafiados u otros de esta naturaleza, como los 'solaque', o cuando sirven de soporte a alguna ornamentación pictórica o una pintura mural. En estos casos se emplea el procedimiento de consolidación por inyección, que es un proceso delicado que debe ser ejecutado por personal especializado, aún más cuando se trata de murales, donde es una fuerte obligación que intervenga un restaurador de bienes inmuebles calificado o un arquitecto con experiencia en arquitectura patrimonial.

Los pasos de este procedimiento pueden tener variantes según el estado en que se encuentre el revoque y dependen del criterio del restaurador. En primer lugar, se procede a detectar la zona de oquedad por el procedimiento de percusión, que consiste en golpearla con un trozo pequeño de madera o con los nudillos, para delimitar el área afectada; posteriormente el revoque se perfora con un taladro manual, usando una broca de $1 / 16$ ", y luego se efectúa la inyección de la lechada de cal con una jeringa de veterinario, siempre con la precaución de cerciorarse periódicamente de que el líquido penetre adecuadamente. A veces, cuando el revoque presenta determinadas características de grosor o de relieve, se puede fijar a la perforación una boquilla o una pipeta por la que se inyecta la lechada.

Antes del uso generalizado en los aditivos modernos, las lechadas para este tipo de inyecciones se componían con aglutinantes como la caseína, el blanco de España, los talcos, entre otros; en la actualidad se emplean los acetatos de polivinilo, los acrilatos y metacrilatos adicionados con una carga de cal cernida. Por lo tanto, todos estos procesos deben ser llevados a cabo por personal especializado y el uso de diversas sustancias varía no solo de acuerdo al restaurador, sino a la región.

Es importante destacar que en cualquier obra de intervención se debe proceder a detectar si es que hay revoques singulares como el solaque hecho a base de cal, arcilla y ladrillo molido. Algunos estudiosos aseguran que durante largo tiempo se utilizó sangre de toro; no es así en todos los casos, y muy comúnmente estos vestigios han quedado debajo de aleros y balcones, o en las jambas de los vanos.

Sin embargo si se desea dar protección a los revoques se debe aplicar productos hidrofugantes por aspersión o brocha; estos evitan la entrada del agua a las capas más profundas y al muro. El hidrofugante debe tener como característica permitir 'respirar' al revoque, pero también evitar la penetración del agua. Además, se recomienda el uso de hidrofugantes hechos de silanos xiloxanos (Orías, 2011). 


\section{Pisos}

Por lo general, en las casas patrimoniales existen ladrillos pastelón y baldosas hidráulicas, llamadas también mosaicos; también hay pisos de madera machihembrada y, en excepcionales casos, de tipo parquet. En cada caso se debe seguir pautas específicas, porque las características de los materiales son diferentes. Los pisos de la sala, donde quedaba el lobby, también llaman la atención: presentan un diseño con baldosas hexagonales de colores negro, gris claro y rojo óxido; en los demás ambientes presenta baldosas cuadradas con un patrón a modo de damero en negro y gris claro.

El corredor del pasillo está recubierto de ladrillo pastelón en tono gris; si un piso equivalente se encuentra en buen estado, debe procederse a un mantenimiento periódico que consista, de manera simple, en una limpieza con un cepillo de cerdas suaves y detergentes neutros, aunque en otros tiempos se empleaba el aceite de linaza para combatir la porosidad, consolidar las piezas y darle mayor brillo (Figura 15).

En caso las piezas posean un valor artístico o las piezas estén erosionadas, se debe aplicar consolidantes como el silicato de etilo, que se aplican por aspersión. El Paraloid es utilizado localmente para este fin; sin embargo, es menos recomendable que el producto antes mencionado. Por otro lado, cuando la pieza está rota o en estado de deterioro grave, es más conveniente sustituirla por otra pieza en buen estado. De esta manera se evita el deterioro al interior del forjado, ya sea por la infiltración de agua o la acumulación de suciedad en los huecos, lo que a su vez puede provocar el desprendimiento de las piezas vecinas. En el caso del ladrillo del exterior de la casa Fariñas, se encuentra en buen estado, aun si se considera que está a la intemperie.

Las baldosas hidráulicas son muy comunes en las casas patrimoniales, y sus estilos van desde los más simples, como los que están en la casa Fariñas, hasta los más intrincados, con diseños florales y geométricos. Por lo tanto, para la limpieza de este tipo de las baldosas en general se debe utilizar detergentes neutros y evitar todo tipo de ácidos, pero, si fuese necesario, se puede utilizar hipoclorito de sodio (lavandina) o amoniaco para la eliminación de manchas. En ese caso, siempre debe tenerse la precaución de enjuagar las baldosas con agua después de aplicarlo para neutralizarlo, y así evitar los encharcamientos (Figura 16).

En principio este material no requiere de ningún tipo de protección; no obstante, por las diferentes agresiones que haya podido sufrir, o por el desgaste por el tránsito diario, puede plantearse la necesidad de protegerlos, y devolverles el aspecto perdido. Para ello debe recurrirse a la aplicación de cera natural, pero nunca plástica, con la recomendación de que esta operación se realice con una pulidora para conseguir una mayor saturación y una superficie más uniforme.

En Sucre, al igual que en Masaya, el uso del hierro forjado y fundido se remonta a los primeros años de la ciudad; sin embargo, fue en el siglo XIX y principios del XX cuando más se difundió su utilización. De este tiempo datan la mayoría de las construcciones con puertas de vasistas y puertas de hierro forjado, como en el caso del antiguo hospital -hoy hogar asilo San Antonio-. Los elementos más simbólicos del arte de la forja en la ciudad son las rejerías que forman parte de los balcones, ventanas, montantes de puertas o vasistas; en las puertas tipo cancel de los zaguanes también existen elementos como llamadores o tocadores, clavos con cabeza decorada (tetones), bisagras y otros elementos de herrería (Orías, 2011).

Para todos los casos, los tratamientos de mantenimiento y protección son los mismos, y la carpintería de hierro requiere de cuidados específicos; su ubicación habitual en las fachadas o corredores la expone a la lluvia, el frío y otras inclemencias producto de la intemperie, que los estropean con el paso del tiempo. Algunos sencillos cuidados de limpieza y un tratamiento protector periódico ayudan a evitar el deterioro. Como
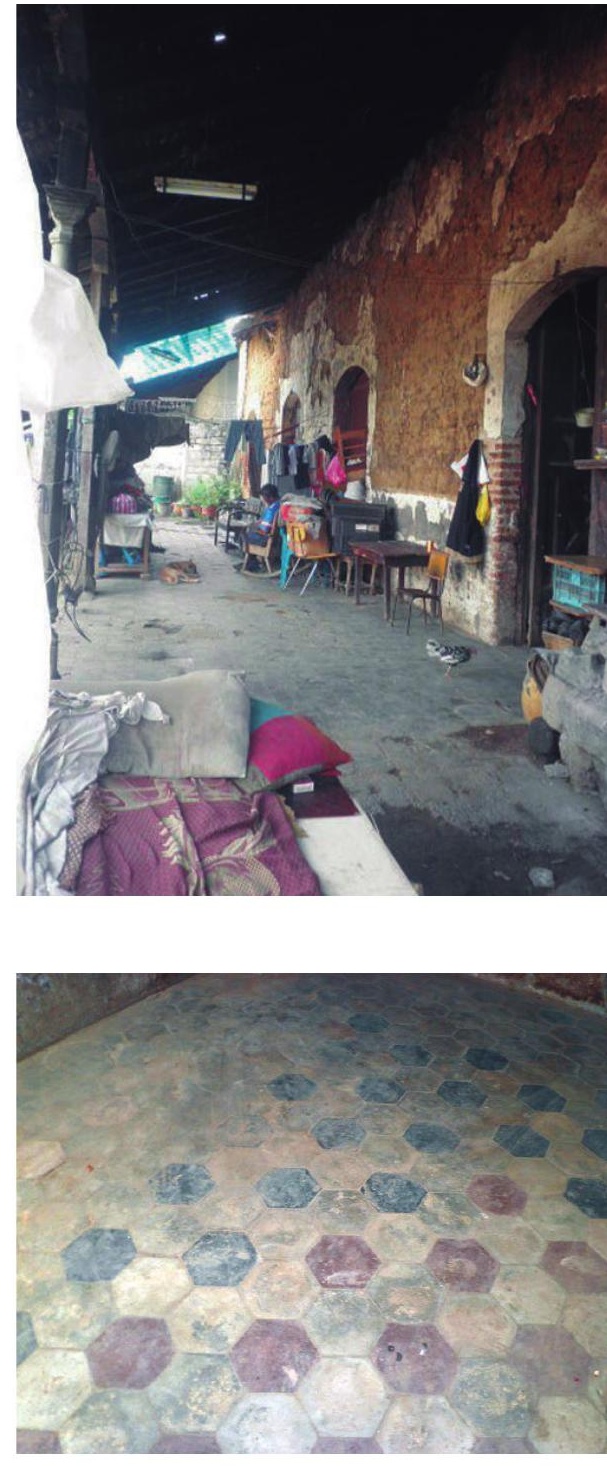

Figura 15. Corredor con ladrillo pastelón en tono gris

Fuente: Archivo fotográfico Nohemí Balmaceda, 2015.

Figura 16. Piso de baldosas hexagonales en tres colores: gris claro, negro y rojo óxido

Fuente: Archivo fotográfico Nohemí Balmaceda, 2015. 
se sabe, el hierro se caracteriza por su estabilidad y resistencia al paso del tiempo; sin embargo, esta durabilidad no siempre garantiza un buen aspecto. La oxidación es uno de los principales enemigos de estos elementos, que deben protegerse con una capa de pintura o barniz antioxidante.

Otra opción es realizar un mantenimiento periódico con una cera especial para metales, ya que este producto combate la corrosión y evita los efectos de agentes externos sobre las piezas. En caso se requiera restaurar el metal, se debe seguir un procedimiento que se basa en limpiar una pieza de hierro forjado empleando pocas herramientas. Es suficiente usar un cepillo de hierro o carda, y, de forma opcional, un rodillo de púas metálicas en el taladro, que servirá para quitar la pintura y el óxido que puede tener la pieza a restaurar; en algunos casos también es necesario utilizar disolventes. No obstante, cuando el elemento tiene restos antiguos de pintura, lo más adecuado es utilizar un decapante para eliminarlos, puesto que el decapante reblandece la pintura o barniz, y permite quitarla más fácilmente con una espátula y retirar el polvo que se forma con un paño, para luego estudiar a detalle la pieza para comprobar que esté completa $y$, en caso le faltara una parte principal o un elemento decorativo, intentar saber o intuir, por medio de un levantamiento y una catalogación del elemento, qué falta y cómo debe ser su correcta reposición. Además, se puede recurrir a viejas fotografías, en el caso de encontrarlas (Orías, 2011).

Una vez que se conoce qué elementos faltan, se elabora una plantilla, lo más parecida posible a la pieza a reponer, que permita dibujar, recortar y rectificar, hasta alcanzar la forma y medida adecuada. Una vez hecha, la plantilla se acopla a la pieza en estado de restauración y se procede al forjado de la pieza en hierro. El siguiente paso es la fijación de las piezas nuevas a través de métodos reversibles y respetuosos, de forma que quede lo más integrada posible sin que se perciba que es un elemento añadido. Para el procedimiento se aconseja prudencia y no pretender acabar pronto; en este caso el proceso suele ser conciso y pausado por tratarse de trabajos delicados. Una vez que la pieza está completamente limpia, y con los elementos que antes faltaban ya repuestos, se procede a darle una capa de minio o imprimación antioxidante para que, una vez seca, dicha capa se pueda pintar.

En cuanto a la pintura que se debe usar para una casa patrimonial, es crucial tomar en cuenta que las pinturas originales están elaboradas a base de pigmentos naturales que son totalmente compatibles con los revoques de barro y muros de adobe, ya que la cal permite la 'respiración' de los mismos. Por esta razón, la pintura tradicional con agua de cal o las pinturas contemporáneas en base a agua son las opciones que se pueden elegir en los acabados de muros de tierra. Siempre debe evitarse emplear pinturas de aceite, ya que estas no permiten la transpiración de los muros y revoques, lo que ocasiona el ingreso de humedad y sales hacia el interior. Para la aplicación de color, se deberá proceder en primera instancia con calas de prospección con el fin de conocer el color original de la superficie. Posteriormente se retira la capa superficial de polvo y suciedad con la ayuda de una lija suave y, en caso existan capas de pinturas de aceite, estas deben ser eliminadas totalmente, puesto que su presencia no permitirá la adherencia de la nueva capa de pintura. En caso las calas de prospección revelen la existencia de pintura mural se debe requerir los servicios de un especialista restaurador en pintura (Orías, 2011).

\section{Conclusiones}

La ciudad de Masaya es símbolo de la cultura popular de Nicaragua por la vitalidad de sus costumbres y tradiciones, y por ser una ciudad auténtica y llena de historia gracias a la cotidianidad de sus pobladores y los escenarios de sus manifestaciones culturales.

La ciudad posee importantes valores culturales, históricos, económicos, artísticos, turísticos, ambientales, urbano-arquitectónicos, entre otros; estos valores están conte- 
nidos y expresados en los atributos que la hacen ser poseedora de grandes atractivos patrimoniales que podrían ser potenciados para el turismo, de tal forma que beneficien a la población en general.

La normativa no solo implica regulaciones que restringen la protección del patrimonio cultural, sino que busca concientizar, apropiarse del patrimonio y disfrutarlo, y dar apoyo a la regulación de propuestas y proyectos que resguarden el patrimonio cultural para beneficio de los pobladores.

El centro de la ciudad es donde se desarrollan la mayoría de las manifestaciones culturales, donde se encuentran los inmuebles de mayor relevancia, donde las personas se apropian y viven su identidad como ciudadanos, y donde estos mismos producen y renuevan la cultura. Al consolidar completamente esta área, y regular el manejo y las intervenciones que en ella se proyectan, la población se apropiará de su historia y la riqueza urbanística patrimonial, y, por consiguiente, se tendrá mayor conciencia respecto de la protección de estos inmuebles.

La ciudad de Masaya aún es una ciudad auténtica por conservar su patrimonio cultural, en particular el inmaterial. Su patrimonio construido es de interés por sus monumentos emblemáticos y su arquitectura vernácula que aún no ha sufrido de manera descontrolada el abandono de las viviendas por parte de sus dueños tradicionales para ser adquiridas por foráneos, como ha ocurrido en muchos centros históricos en América Latina. Sin embargo, no se descarta la posibilidad de que ocurra este fenómeno, donde la arquitectura original se desvirtúa a través de ampliaciones, remodelaciones o restauraciones, que terminan en la destrucción del espacio y la falsificación de fachadas. En otros casos las fachadas son tiradas, y se hacen réplicas de las mismas con el uso de materiales y decoraciones que no tienen ninguna relación, o aproximación, con los originales, y que dan paso a la perdida de las tradiciones y la convivencia comunitaria, y convierten a la ciudad en un parque temático (Secretaría Técnica de la Alcaldía de las Familias y Comunidades de Masaya, 2012).

Cuando la electricidad y la telefonía llegaron a Masaya, lejos de traer el progreso que se esperaba trajeron consigo el indeseable cableado que deteriora la imagen urbana de toda la ciudad. Incluso hay parques por donde aún cruzan de lado a lado cables de media y baja tensión, que deforman la imagen de las fachadas de casas históricas como la de la familia Fariñas. Esto provoca que, por la agresión que significa la reciente existencia de estas instalaciones, disminuya su esplendor, pero ellas son absolutamente necesarias para la vida contemporánea, por lo cual no se pueden dejar de lado. El entierro de la red aérea de baja y media tensión le compete al gobierno municipal, y a las instituciones encargadas de servicios urbanos, a los cuales se debe concientizar para contribuir al embellecimiento de la imagen del centro histórico de Masaya. Mientras se soterran las redes, existen varias opciones para mejorar el aspecto de las fachadas con cableados grapados. Además, los cables se pueden pasar por una tubería rígida interior con registros de entrada y salida en ambos extremos de las fachadas; se puede considerar fijar un ducto provisional que recoja todos los cables y pueda ser pintado del mismo color de la fachada, y sujetar este ducto por encima o por debajo de una cornisa, friso o moldura para mimetizarlo con la fachada (Orías, 2011).

Las fachadas cuidadas de las viviendas constituyen el rostro de una ciudad que, en busca de un progreso caótico, no escatima en la protección de los bienes patrimonio. Este rostro es fundamental para la imagen urbana del centro histórico, pero las constantes intervenciones, sean buenas o malas, diariamente se plasman sobre estas fachadas, cuyo carácter histórico esta dado a razón de su arquitectura, sus ornamentos y su estética, así como su entorno urbano. Además, estas intervenciones, aunque parezcan casos inofensivos que se dan de forma aislada, con el tiempo, de manera conjunta, logran no solo modificar el inmueble, sino todo su entorno para siempre. Se transforman en un peligro para la supervivencia de la imagen tradicional de la arqui- 
tectura doméstica, la cual, por este calificativo, muchas veces se deja en el olvido. Por ello, este artículo esboza un resumen de no solo el daño de esta vivienda, que es parte del patrimonio de Masaya, sino de la solución que debería ser aplicada para su restauración y para la conservación de las características del elemento tradicional u original. Se plantearon por partes las posibles intervenciones, y se valoraron los inmuebles, así como la calidad de imagen urbana de la ciudad, de manera cuidadosa para llevar a cabo la restauración del elemento con el mayor grado de calidad posible. Son innumerables los elementos y ornamentos que enriquecen las fachadas, y cada uno es digno de estudio, por lo cual en todo el artículo se hizo hincapié en los elementos más comunes y los de mayor aporte en la imagen urbana de un centro histórico. El cuidado con el que el proyectista y el constructor, con su equipo especialista, intervienen en un proyecto sobre un bien patrimonial se refleja siempre en los acabados de cada parte de la casa, desde la estructura hasta los ornamentos.

Es deber de todos los ciudadanos de Masaya, así como del gobierno municipal, pero más aun de los dueños de viviendas históricas, no solo reconocer su valor y los detalles que logran que destaquen entre otras con la misma data y características similares -eso que las hace únicas y diferentes-, sino darles el debido mantenimiento para conservar bienes que son parte del acervo cultural de la hermosa ciudad de Masaya.

La conservación y recuperación de las técnicas y materiales tradicionales forman parte del acervo cultural único y específico de cada comunidad, y, por tanto, se los debe estudiar y proteger (Orías, 2011). Por esta razón es importante no dejar que bienes culturales inmuebles como la casa de la familia Fariñas desaparezcan; son parte de la memoria de la ciudad, y, a pesar de su aspecto y del común rechazo por parte de los transeúntes, estos bienes culturales inmuebles no solo deben ser apreciados por las personas usuarias de las mismas, sino por su entorno y por los ciudadanos. Es una lástima que los funcionarios municipales, encargados de velar por el acervo cultural de una ciudad tan representativa de la cultura nicaragüense, que tiene 177 años de haber sido elevada a la categoría de ciudad y que ha sido nombrada cuna del folklor, vean caer frente a sus ojos la cultura de una ciudad. Es entonces necesario concientizar a todas las personas de la importancia de resguardar y conservar el patrimonio.

\section{Referencias}

Alcaldía de Masaya. (Septiembre de 2012). Clasificación de inmuebles y espacios urbanos. Números catastrales. Masaya, Nicaragua: Autor.

Alcaldía de Masaya. (Septiembre de 2012). Normativa del área patrimonial de la ciudad de Masaya. Masaya, Nicaragua: Autor.

Instituto Nicaraguense de la Cultura. (2009). Cartilla para propietarios y gestores culturales de Bienes Culturales Inmuebles. Managua, Nicaragua: Autor.

Orías, L. P. (2011). Manual para la conservación del patrimonio arquitectónico de Sucre. Conservando nuestro patrimonio, I(1), 97.

Secretaría Técnica de la Alcaldía de las Familias y Comunidades de Masaya. (Agosto de 2012). Delimitación del área patrimonial de la ciudad de Masaya [borrador]. 